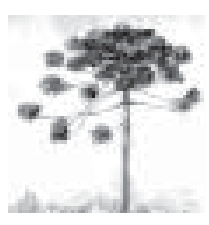

\title{
O CONHECIMENTO DE RECURSOS ALTERNATIVOS EM PESQUISA COM ANIMAIS DE LABORATÓRIO
}

\author{
A lternative resources knowledge in laboratory animals research
}

\author{
Letícia Meira Pinheiro', Luiz Antônio Acra² \\ ${ }^{1}$ Bióloga, Bacharel; Pontifícia Universidade Católica do Paraná; CCBS; Herbário HUCP. Curitiba, PR - Brasil, \\ e-mail: tici9@ yahoo.com.br \\ ${ }^{2}$ Biólogo, Especialista; Pontifícia Universidade Católica do Paraná; CCBS; Herbário HUCP. Curitiba, PR - Brasil, \\ e-mail:luiz.acra@ pucpr.br
}

\begin{abstract}
Resumo
0 presente artigo tem por objetivo levantar dados sobre a questão da utilização de recursos alternativos em substituição aos animais de laboratório, bem como averiguar o nível de conhecimento e a possibilidade de utilização desses meios por parte de professores, alunos e pesquisadores da Pontifícia Universidade Católica do Paraná. Constatou-se que apenas 40\% dos entrevistados possuem conhecimento sobre recursos alternativos. O bservou-se ainda que $53 \%$ dos entrevistados veem a possibilidade do uso desses recursos em seus experimentos. Entre os pesquisadores que já utilizaram ou utilizam os recursos alternativos, $67 \%$ considera os resultados obtidos adequados em comparação aos experimentos realizados com cobaias animais.
\end{abstract}

Palavras-chave: Bioética; Recursos alternativos; Animais de laboratório.

\begin{abstract}
The purpose of this present article is to raise data about the use of alternative resources in substitution to laboratory animals, as well as inquiring the knowledge level and possibility of using these resources by professors, students and researchers of Pontifícia Universidade Católica do Paraná. It was observed that only $40 \%$ of the interviewed people had knew about alternative resources. It was also observed that 53\% of the interviewed people see the possibility of using these resources in their own experiments. Between researchers that had already used or still use alternative resources, $67 \%$ consider the gotten results adequate in comparison to the experiments carried through with animal guinea pigs.
\end{abstract}

Keywords: Bioethics; Alternative resources; Laboratory animals. 


\section{INTRODUÇÃO}

A utilização de animais na tentativa de compreensão de processos biológicos começou há muitos anos com Hipócrates (450 a.C.) que, didaticamente comparava 0 aspecto de órgãos humanos doentes com o de animais. Galeno (129 210 d.C.), em Roma, foi talvez o pai da medicina científica, realizando procedimentos experimentais semelhantes à vivissecção, testando algumas variáveis em modelos animais. Estudou também a anatomia e fisiologia de macacos acreditando na similaridade com o homem (1).

Entretanto, o uso de animais em experimentos sempre foi alvo de muitas críticas por vários segmentos da sociedade, questionando 0 sofrimento dos animais após manipulacõos. 0 filósofo RenéD escartes (1596-1650 d.C.) acreditava que os processos de pensamento e sensibilidade faziam parte da alma. Como em sua concepção os animais não tinham alma, não havia sequer a possibilidade de sentirem dor. Claude Bernard, em 1860, dizia que é parte da postura do cientista ser indiferenteao sofrimento dosanimais delaboratório. Posteriormente, em 1865, justifica a utilização de animais em pesquisa alegando que seria estranho utilizar animais para serviços caseiros, para comida, e proibir seu uso para ensino e pesquisa (1).

Um movimento mundial busca alternativas para o uso de animais em experiências, propondo sua substituição por outros recursos técnicos, como culturas celulares in vitro, modelos computadorizados, simuladores e até placenta humana, usada para praticar cirurgia microvascular (2). Segundo Lopes (2), a polêmica não se baseia mais numa atitude meramente sentimental e caridosa em relação às cobaias indefesas, luta-se contra essa ciência ultrapassada, que ainda faz uso de animais em suas pesquisas, o que leva a resultados pouco confiáveis.

No Brasil há um movimento próalternativas que se divide em duas linhas de ação: uma apoia o controle da experimentação animal através de legislacão específica e comitês de ética, a outra se bate pela abolição total de tal prática (2).

\section{A legislação sobre experimentação animal}

Foram, também, levantados dados a respeito da legislação. Constatou-se a existência de leis específicas sobre a experimentação animal no Brasil, entretanto, não existe uma jurisdição exclusiva sobre o assunto. A Lei 6.638/79 estabelece normas para a vivissecção em todo 0 país, desde que essas atividades didático-científicas sejam realizadas no ensino superior e não causem sofrimento aos animais, porém isso não ocorre na maioria das faculdades brasileiras (2).

A Lei de Crimes Ambientais, Lei $\mathrm{n}$ 9605 de 12 de fevereiro de 1998 representou um grande avanço, transformando em crime, a crueldade e os maus-tratos em animais. 0 parágrafo $1^{0}$ do artigo 32 da referida lei, adotando parcialmente as noções contidas nos três " $\mathrm{R}$ 's"*, estipulou a pena de detenção de três meses a um ano e multa, para "quem realiza experiência dolorosa ou cruel em animal vivo, ainda que para fins didáticos ou científicos, quando existirem recursos alternativos" (3).

A comunidade científica brasileira clama, pois, por uma sistematização definitiva e por uma normafederal abrangente e realista, mais orientadora e reguladora, e menos sancionadora, onde a questão da pesquisa científica possa ser mais bem contemplada (3).

É importante saber do conhecimento de pesquisadores e cientistas sobre as outras opções para a realização de seus trabalhos, como modelos biológicos alternativos, bem como lhes apresentar suas vantagens e desvantagens, para que assim, algumas vidas animais que hoje estariam sendo exterminadas sem necessidade, sejam poupadas.

Este trabalho tem por objetivo levantar e analisar dados sobre a utilização de animais em pesquisas laboratoriais, por parte de professores, alunos e pesquisadores da Pontifícia Universidade Católica do Paraná, assim como, seu nível de conhecimento e sobre a possibilidade de utilização de recursos alternativos para a pesquisa com animais em laboratório.

\footnotetext{
Trata-se de um conjunto de recomendações para o uso humanitário de animais em laboratórios feito pelos cientistas ingleses Willian Russel e Rex Burch, hoje mais conhecidas como os três R's: replacement, substituição de animais por técnicas alternativas; reduction, redução do número de animais usados nos experimentos; e refinement, refinamento das técnicas, como, por exemplo, treinamento das pessoas que vão trabalhar com as cobaias (2).
} 


\section{MATERIAL E MÉTODOS}

O trabalho iniciou com um levantamento bibliográfico sobre o assunto em questão, buscando ainteração sobre osmodelos biológicosalternativos, quais são eles, como funcionam, onde se daria sua melhor aplicação, em que campo e suas vantagens e desvantagens em relação ao uso de animais propriamente ditos, além de procurar saber sua viabilidade econômica e de infraestrutura perante as necessidades dos usuários de cobaias animais.

As informacões foram obtidas através de um instrumento de pesquisa (ANEXO I). Juntamente a este foi anexada uma tabela (ANEXO II) contendo os principais métodos biológicos alternativos e seu respectivo detalhamento. 0 instrumento de pesquisa foi submetido ao Comitê de Ética em Pesquisa com Seres Humanos da Pontifícia Universidade Católica do Paraná, tendo obtido sua aprovação através do ofício 338/ 05/ CEP - PUCPR, de 09 de setembro de 2005.

As entrevistas foram realizadas na Pontifícia UniversidadeCatólica do Paraná, Campus de Curitiba, com alunos, estagiários, professores e pesquisadores, que utilizam ou que já tenham utilizado animais em suas pesquisas. Foram realizadas 60 entrevistas no total.
Após a coleta de dados, as respostas foram tabuladas e analisadas através da construção de tabelas e gráficos demonstrativos, em programa MICROSOFT ® Excel 2002, que serviram como base para a obtenção de resultados e conclusão final.

\section{RESULTADOS E DISCUSSÃO}

Ao analisar as entrevistas, pôde-se notar que a maioria do uso de animais dentro da Pontifícia Universidade Católica do Paraná, se dá para fins de pesquisa, chegando a $67 \%$ do uso total. 0 rato mostrou-se como o animal mais utilizado, com um volume de $44 \%$ de uso, seguido pelo camundongo, com $20 \%$, invertebrados com 8\%, cachorros com $6 \%$, porcos, coelhos e peixes com $4 \%$, e rã, aves, bovinos e ovinos com 2\% (Figura 1). Segundo Pimenta e Silva (4), essa preferência por ratos e camundongos se dá em grande parte pelo pequeno porte do animal e também pelo fácil acesso a grandes quantidades desses animais. Tendo em vista a existência de um biotério próprio na PUCPR, 0 acesso e facilidade de obtenção em quantidades significativas desses animais tornam-se maior ainda.

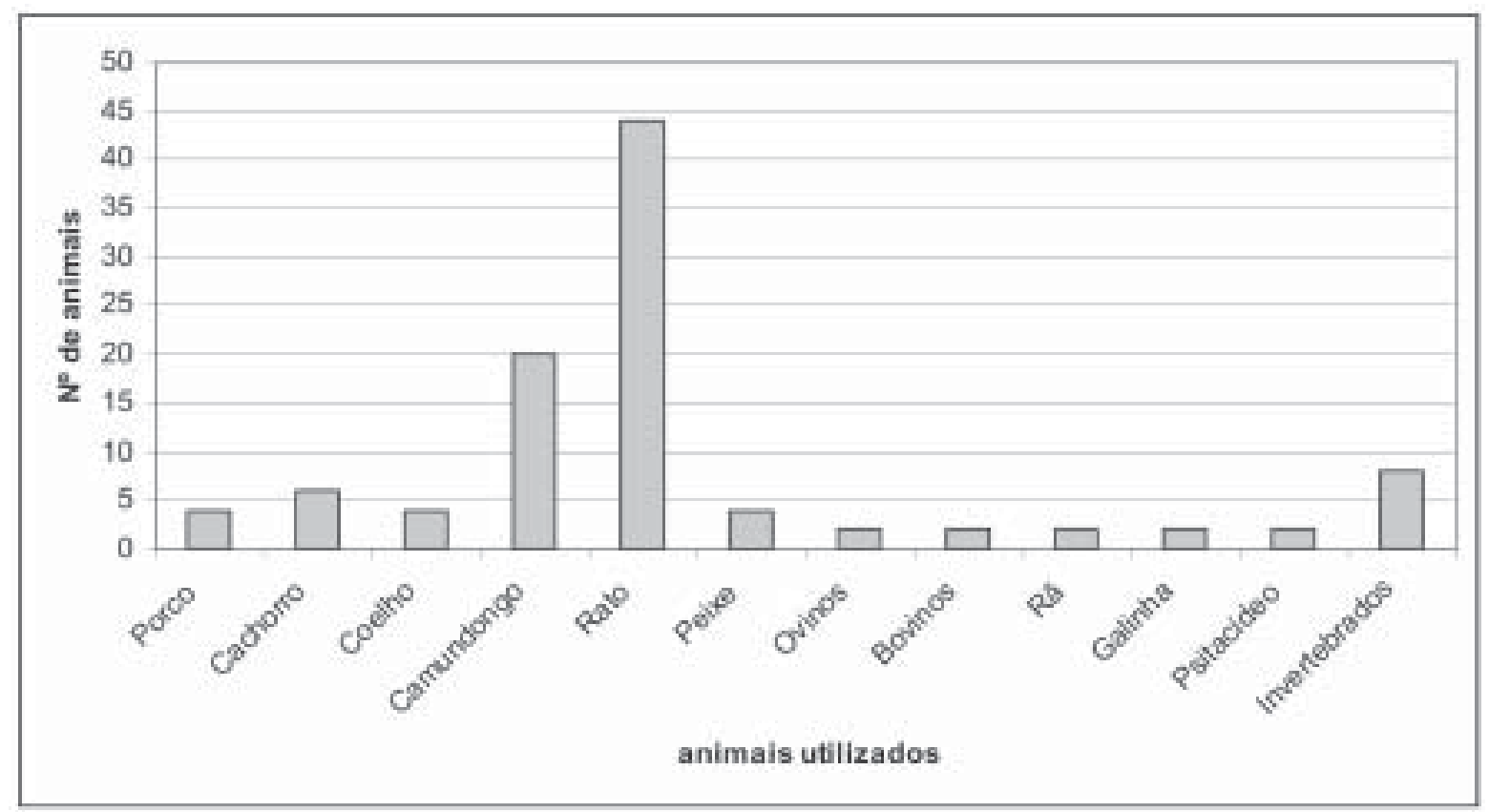

FIGURA 1 - G ráfico de quantificação dos animais utilizados em pesquisas 
Segundo Fagundes e Taha (5), num levantamento nas bases de dados da Biblioteca Regional de Medicinaincluindo a Medline (National Library of Medicine- USA), Lilacs (LiteraturaLatino Americana e do Caribe em Ciências da Saúde), SciELO (Scientific Electronic Library Online) e Biblioteca Cochrane (The Cochrane Database of Systematic Reviews) sobre as seis espécies animais mais comumente citadas, num período de quatro anos, mostrou que os ratos são os animais mais usados em pesquisas, com índices percentuais que variam entre $36,3 \%$ e 43,3\% em todas as bases de dados pesquisadas, seguidos pelos camundongos, com porcentagens entre $21,1 \%$ e $41,7 \%$.

A maior parte dos entrevistados, 93\%, possui conhecimento sobre as normas éticas na utilização de animais em experimentos, e que esse conhecimento foi adquirido, em $57 \%$ dos casos, em cursos de graduação e/ ou pós-graduação; o que mostra que as entidades de ensino, e seus pesquisadores e alunos, ao utilizarem animais em seus experimentos, reconhecem a importância do conhecimento dessas normas, para que a utilização não se dê com sofrimento.

Com relação aos recursos alternativos existentes, o conhecimento dos entrevistadosalcançou um gradiente relativamente considerável, tendo em vista que quase metade destes conhece tais recursos, alcançando um percentual de 40\% do total (Figura 2).

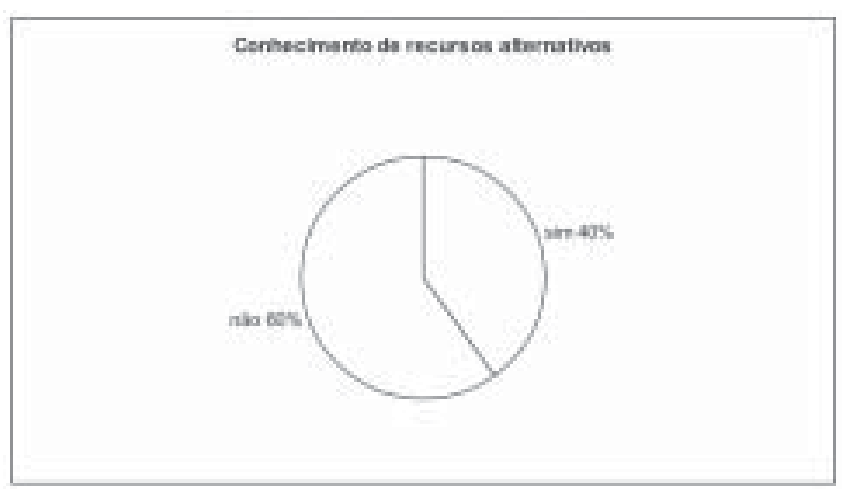

FIGURA 2 - G ráfico sobreo conhecimento dosRecursos Alternativos

Porém, com relação aos entrevistados que afirmam conhecer, apenas 13\% utilizam esses recursos de fato. Dos entrevistados que ainda não lidam com os recursos alternativos, 53\% consideram a possibilidade de usar esses recursos futuramente em suas pesquisas (Figura 3).

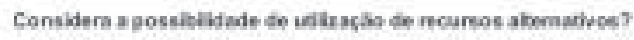

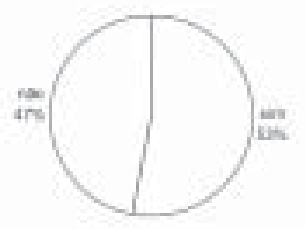

FIGURA 3 - G ráfico sobre a possível utilização dos Recursos Alternativos

Em substituição aos animais, os recursos alternativos mais citados pelos pesquisadores como passíveis de utilização foram a cultura celular e a cultura tissular, principalmente em virtude de tais métodos caracterizarem-se pelo fácil acesso em laboratórios, entretanto outros modelos também obtiveram citações, dentre eles: a utilização da placenta, da informática, e de pesquisas clínicas e epidemiológicas.

Alguns fatores que dificultam ou até impedem o uso desses recursos alternativos são: a questão financeira e 0 fato de alguns pesquisadores não considerarem os resultados obtidos adequados em comparação aos experimentos tradicionais. A questão financeira foi mencionada pelos entrevistados, completando que até consideram a possibilidade de usarem recursos alternativos, mas que nem sempre estes estão disponíveis pelo fato de serem mais caros que as tradicionais cobaias animais, já criadas no biotério daPUCPR. Quanto aos resultados, muitos pesquisadores consideram que os animais seriam dispensáveis e que os resultados obtidos com a utilização de recursos alternativos seriam adequados, de acordo com $67 \%$ dos entrevistados (Figura 4), entretanto, alguns pesquisadores ainda consideram que apenas eles, os animais, podem ser eficientemente usados, principalmente em cirurgias e em experimentos de fisiologia e neurociências, por exemplo. D entre alguns relatos feitos pelos entrevistados, foi citado que os resultados quando da utilização de recursos alternativos são muito superficiais e que, dependendo do objetivo da pesquisa, não seriam resultados satisfatórios. 


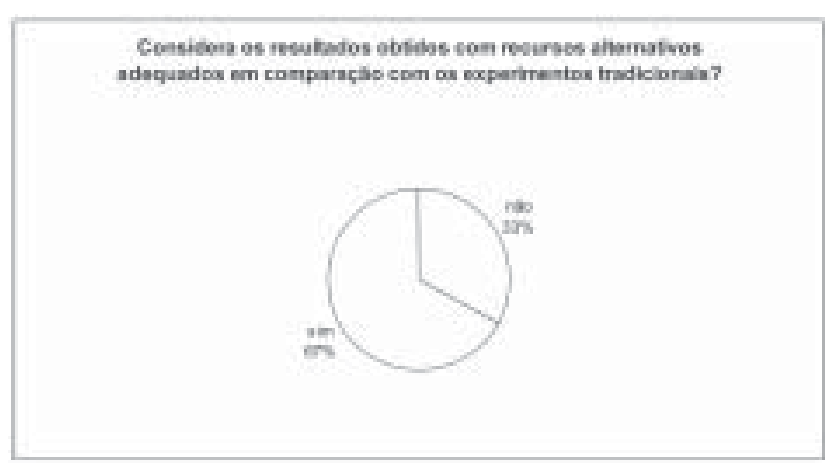

FIGURA 4 - Comparação entre os resultados obtidos com recursos alternativos e experimentos tradicionais

Segundo Marques et al. (3), modelos desenvolvidos em computadores ou órgãos isolados ainda apresentam grande limitação e, em grande parte, não podem ser consideradas alternativas satisfatórias. Entretanto, alternativas viáveis e substitutivas a seu uso devem ser continuamente buscadas. Os testes toxicológicos ainda utilizam animais porque até 0 momento não existem métodos alternativos validados para 0 exame de inocuidade: 0 FDA (Food andD rugs Administration)aindaconsidera o método D raize (ANEXO I) como o mais confiável para avaliação de segurança de cosméticos (2).

\section{CONSIDERAÇÕES FINAIS}

A substituição total dosanimais, infelizmente ainda é uma utopia, pois em vários procedimentos ainda são de extrema importância, sendo através deles o único meio de se chegar a resultados mais precisos. É por esse motivo que os animais ainda são tão utilizados nos processos de pesquisas científicas e, como o conhecimento a respeito dos recursos alternativos que se tem hoje em dia é relativamente pequeno, os animais continuam sendo indispensáveis a essas práticas. Portanto, é importante frisar que, quando o uso decobaiasanimaissefizerindispensável, a principal questão é respeitar a condição de fazer uso somentede número suficientepara seobter aresposta, de não deixá-los sofrer, de empregar a sedação, analgesia e anestesia necessárias e adequadas (3). Segundo Aguilar-Nascimento (6), o importante para a preservação de vidas animais, evitando-se usos dessas vidas desnecessariamente, é que o projeto a ser realizado defina muito bem qual é a informação visada, ou seja, o pesquisador deve definir qual é a pergunta a ser respondida com 0 experimento.

\section{REFERÊNCIAS}

1. Pompeu E, Gimenez MP, Camargo RS. Medicina legal e ética experimental. Revista Brasileira de Medicina Legal [online]. 2004 [acesso 2004 nov. 22];Ano 2(4) Disponível em: http:/ / www.revistademedicinalegal.com.br

2. Lopes CF. Em defesa das cobaias. As ONGs protestam: os bichos sofrem nos laboratórios e isso não é necessário. Revista $\mathrm{G}$ alileu [online]. 2004 [Acesso nov. 22]; p. 104. Disponível em: http:/ / www.pfilosofia.pop.com.br/ 04 11_galileu_07.html

3. Marques RG, Miranda ML, Caetano CER, Biondo-Simões ML. Rumo à regulamentação da utilização de animais no ensino e na pesquisa científica no Brasil. A cta Cir Bras. [online]. 2005 [Acesso 2004 nov. 02];20(3):2533. D isponível em: http:/ / www.scielo.br/ abc

4. Pimenta LG, Silva AL. Ethics and animal research. A cta Cir Bras. [online]. 2001 [Acesso 2005 nov. 05];16(4): 55-67. D isponível em: http:/ / www.scielo.br

5. Fagundes DJ, Taha MO. Animal disease model: choice's criteria and current animals specimens. Acta Cir Bras. [online] 2004 [acesso 2005 nov. 02];19(1):19-23. Disponível em: http:/ / www.scielo.br

6. Aguilar-Nascimento JE. Fundamental steps in experimental design for animal studies. Acta Cir Bras. [online] 2005 [acesso 2005 out. 29];20(1):40-49. D isponível em: http:// www.scielo.br/ abc

7. Bernardi S. Projeto de Lei n $\cong$ 1.691, de 2003. Dispõe sobre o uso de animais para fins científicos e didáticos e estabelece a escusa de consciência à experimentação animal [online]. [A cesso 2004 nov. 22]. D isponível em: http:/ / w w w. f o r u m nacional. com . br / pl_1961_03_com_exp_form.pdf

Recebido: 06/ 02/ 2007

Received: 02/ 06/ 2007

Aprovado: $25 / 03 / 2007$

A pproved: 03/25/ 2007 


\section{ANEXO I}

Os principais experimentos realizados com animais de laboratório são:

Vivissecção - cirurgias feitas em animais vivos, anestesiados ou não, para pesquisas médicas, científicas, laboratoriais e didáticas. A prática possibilita o estudo de processos biológicos ou a fenomenologia de enfermidades. $\mathrm{O}$ animal pode ser mutilado e mantido vivo para que suas reações sejam observadas (2).

Método D raize - desenvolvido por John D raize, em 1944, é utilizado para testar o grau de irritabilidade de cosméticos, pesticidas e produtos de limpeza nos seres humanos. Para isso, empregam-se coelhos. No D raize Eye Test, pingam-se substâncias químicas diretamente no olho do animal, que, por vários dias, fica imobilizado pelo pescoço e pelas patas em aparelhos de contenção. Só um dos olhos é danificado, o outro fica intacto para servir de comparação. A primeira reação é o lacrimejamento, aos poucos, o olho inflama até chegar à cegueira completa. Inchado e reduzido a um foco purulento, o olho é retirado e submetido a pesquisas fisiológicas e anatômicas. No D raize Skin Test, raspam-se os pêlos dorsais de um coelho e, com um esparadrapo colado e puxado repetidas vezes, retiram-se camadas superficiais da pele para deixá-la mais sensível. Em seguida, respinga-se a substância química que será analisada e cobre-se a área com ataduras. Dias depois, os ferimentos que se formam são então analisados (2).

Teste LD 50 - A sigla significa Dose Letal para 50\% dos animais. É usado para testar medicamentos, cosméticos, pesticidas e produtos de limpeza. As substâncias são testadas diretamente no estômago de um grupo de animais, geralmente cachorros e roedores, através de ingestão forçada. Na primeira dose, usa-se uma quantidade máxima do produto, que vai sendo diminuída progressivamente durante o experimento. O s cães mortos são substituídos por outros. Os animais sentem náuseas, sofrem convulsões, sangramentos, diarreias e queimaduras na mucosa do aparelho digestivo. Q uando sobram apenas 50\% da população de animais, atinge-se 0 que os pesquisadores consideram a quantidade ideal para a prescrição do medicamento na medicina humana (2).

Toxicológicos - Usados para determinar os efeitos adversos causados pela exposição a uma substância química. Os resultados são calculados com base na relação de exposição e resposta quantitativa. No caso da toxicidade alcoólica e de tabaco, aplicam-se doses cada vez maiores dessas substâncias nos animais, até o limite da sua resistência, o que muitas vezes leva à morte (2).

Convêm relacionar aqui, a título exemplificativo, alguns dos mais conhecidos recursos alternativos, segundo o Projeto de Lei n 1.691, de 2003 (7):

1) Sistemas biológicos in vitro (cultura de células, tecidos e órgãos passíveis de utilização em genética, microbiologia, bioquímica, imunologia, farmacologia, radiação, toxicologia, produção de vacinas, pesquisas sobre vírus e sobre câncer);

2) Cromatografia e espectrometria de massa (técnica que permite a identificação de compostos químicos e sua possível atuação no organismo de modo não invasivo);

3) Farmacologia e mecânica quânticas (avaliam o metabolismo das drogas no corpo);

4) Estudos epidemiológicos (permitem desenvolver a medicina preventiva com base em dados comparativos e na própria observação do processo das doenças); 
5) Estudos clínicos (análise estatística da incidência de moléstias em populações diversas);

6) Necropsias e biópsias (métodos que permitem mostrar a ação das doenças no organismo humano);

7) Simulações computadorizadas (sistemas virtuais que podem ser usados no ensino das ciências biomédicas, substituindo 0 animal);

8) Modelos matemáticos (traduzem analiticamente os processos que ocorrem nos organismos vivos);

9) Culturas de bactérias e protozoários (alternativas para testes cancerígenos e preparo de antibióticos);

10) Uso da placenta e do cordão umbilical (para treinamento de técnica cirúrgica e testes toxicológicos);

11) Membrana corialantoide (teste CAME, que se utiliza da membrana dos ovos de galinha para avaliar a toxicidade de determinada substância);

12) Pesquisas genéticas (estudos com D NA humano)

\section{Recursos Alternativos à Pesquisa com Animais}

Há também alguns outros métodos alternativos, segundo a Frente Brasileira para A bolição da Vivissecção (FBAV):

- Técnicas de Imagens não invasivas - CAT (utiliza computadores na reconstrução de imagens tridimensionais do corpo humano através do Raio-X, MRI (Magnetic Resonance Imaging - permite a visualização de imagens detalhadas do interior do corpo humano, sem injeção de substâncias radioativas), PET (Positron Emission Tomograph) e SPECT (Single Photon Emission Computerized Tomograph ) - usados em estudos de doenças cérebrovasculares e distúrbios psiquiátricos.

- EYETEX - Em substituição ao "D raize Eye Test", é o uso de uma proteína líquida que imita a reação do olho humano.

- Simulação Cirúrgica - Manequim D asie (D og Abdominal Surrogate Instructional Exercise), de baixo custo e fácil manipulação, com o qual é possível simular uma cirurgia abdominal (2). 